\title{
Utilization of PET-CT in target volume delineation for three-dimensional conformal radiotherapy in patients with non-small cell lung cancer and atelectasis
}

Li-Jie Yin ${ }^{1 *}$, Xiao-Bin Yu², Yan-Gang Ren ${ }^{3}$, Guang-Hai Gu1, Tian-Gui Ding ${ }^{1}$ and Zhi Lu ${ }^{1}$

\begin{abstract}
Background: To investigate the utilization of PET-CT in target volume delineation for three-dimensional conformal radiotherapy in patients with non-small cell lung cancer (NSCLC) and atelectasis.

Methods: Thirty NSCLC patients who underwent radical radiotherapy from August 2010 to March 2012 were included in this study. All patients were pathologically confirmed to have atelectasis by imaging examination. PET-CT scanning was performed in these patients. According to the PET-CT scan results, the gross tumor volume (GTV) and organs at risk (OARs, including the lungs, heart, esophagus and spinal cord) were delineated separately both on CT and PET-CT images. The clinical target volume (CTV) was defined as the GTV plus a margin of 6-8 mm, and the planning target volume (PTV) as the GTV plus a margin of 10-15 $\mathrm{mm}$. An experienced physician was responsible for designing treatment plans Plan CT $_{\text {and Plan }}$ PET-CT on CT image sets. 95\% of the PTV was encompassed by the $90 \%$ isodose curve, and the two treatment plans kept the same beam direction, beam number, gantry angle, and position of the multi-leaf collimator as much as possible. The GTV was compared using a target delineation system, and doses distributions to OARs were compared on the basis of dose-volume histogram (DVH) parameters.
\end{abstract}

Results: The GTV $V_{C T}$ and GTV and GTV PET-CT exceeded 25\% in 12 (40\%) patients. The GTV PET-CT decreased in varying degrees compared to the GTV $_{C T}$ in 22 patients. Their median GTV PET-CT and median GTVPET-CT were $111.4 \mathrm{~cm}^{3}$ (range, $37.8 \mathrm{~cm}^{3}-188.7 \mathrm{~cm}^{3}$ ) and $155.1 \mathrm{~cm}^{3}$ (range, $76.2 \mathrm{~cm}^{3}-301.0 \mathrm{~cm}^{3}$ ), respectively, and the former was $43.7 \mathrm{~cm}^{3}(28.2 \%)$ less than the latter. The GTV PET-CT increased in varying degrees compared to the GTV $\mathrm{CT}_{\text {in }} 8$ patients. Their median GTV PET-CT and median GTVPET-CT were $144.7 \mathrm{~cm}^{3}$ (range, $125.4 \mathrm{~cm}^{3}-178.7 \mathrm{~cm}^{3}$ ) and $125.8 \mathrm{~cm}^{3}$ (range, $105.6 \mathrm{~cm}^{3}-153.5 \mathrm{~cm}^{3}$ ), respectively, and the former was $18.9 \mathrm{~cm}^{3}$ (15.0\%) greater than the latter. Compared to Plan $\mathrm{CT}$ parameters, Plan PET-CT parameters showed varying degrees of changes. The changes in lung $V_{20}, V_{30}$, esophageal $V_{50}$ and $V_{55}$ were statistically significant ( $P_{S}<0.05$ for all), while the differences in mean lung dose, lung $V_{5}, V_{10}, V_{15}$, heart $V_{30}$, mean esophageal dose, esophagus Dmax, and spinal cord Dmax were not significant ( $P_{S}>0.05$ for all).

Conclusions: PET-CT allows a better distinction between the collapsed lung tissue and tumor tissue, improving the accuracy of radiotherapy target delineation, and reducing radiation damage to the surrounding OARs in NSCLC patients with atelectasis.

Keywords: Atelectasis, PET-CT, Non-small cell lung cancer, Target volume, Three-dimensional conformal radiotherapy

\footnotetext{
* Correspondence: kevin.liu@thepbpc.org

'Department of Radiotherapy, Dalian Central Hospital, Dalian 116033, China

Full list of author information is available at the end of the article
} 
Table 1 Clinical characteristics of patients

\begin{tabular}{ll}
\hline Clinical characteristic & Cases \\
\hline Age & 20 \\
$\geq 60$ years & 10 \\
$<60$ years & \\
Gender & 19 \\
Male & 11 \\
Female & \\
Histological type & 20 \\
Squamous cell carcinoma & 8 \\
Adenocarcinoma & 2 \\
Adenosquamous carcinoma & \\
Tumor location & 21 \\
Central & 9 \\
Peripheral & \\
TNM stage & \\
IIIA & 14 \\
IIIB & 16 \\
\hline
\end{tabular}

\section{Background}

Radiation is one of the important means for the treatment of non-small cell lung cancer NSCLC. In the process of radiotherapy, target area sketch is very important, and it has important significance in the treatment of patients with curative effect and prognosis. Most of the radiation treatment planning system is based on Computed Tomography (CT) image as a target area sketching and dose calculation basis. However, when NSCLC patients have atelectasis or obstructive pneumonia, it is difficult to distinguish the boundaries between incompletely expanded lung tissue and tumor tissue by conventional CT, which often results in inaccurate target delineation. As a consequence, insufficient dose coverage of the target volume or too much damage to normal tissue is caused. The advent of Positron emission tomography-Computed Tomography (PET-CT) can help overcome this problem. PET-CT is a fusion of functional information got by PET and anatomic information got by CT [1-3]. PET-CT can effectively identify the boundary between atelectasis region and lung cancer, make radiation target area precision, avoid unnecessary radiation injury and reduce radiation complications, thereby improving the radiation effect. The studies of Balogh et al. [4]. have shown that PET-CT imaging avoided the CT miscarriage of justice for tumor obstructive pneumonia or atelectasis organization, so that the gross tumor volume (GTV) narrowed, and found the transfer of lymph nodes which were not founded on CT, so that the GTV increased. Hoseok et al. [5] and Wang et al.[6] research results show that based on the PET - CT radiation plan can improve the outline of the GTV, helps to reduce the high dose lung and esophageal illuminated doses, may reduce the lung and esophageal radiation related to toxic reaction and improve the patient's quality of life, and in the same toxic reaction of cases is expected to further improve the target dose, improving the local control rate. The research indicates that the PET-CT improves the accuracy of the clinical stage, provides the basis for patients to choose the correct treatment approach, avoids the treatment select error induced by the mistakes of stage. At the same time, PET-CT can make target area sketch more accurate, better protect surrounding normal tissues, enhance the curative effect and reduce the radiation complications, thereby improving the patient's quality of life (Tables 1, 2 and 3).

In this study, we retrospectively analyzed the PET-CT imaging data for 30 NSCLC patients with atelectasis who underwent three-dimensional conformal radiotherapy and investigated the utilization of PET-CT in target volume delineation in these patients.

\section{Methods}

\section{Clinical data}

Thirty NSCLC patients who underwent PET-CT at the First Affiliated Hospital of Dalian Medical University from August 2010 to March 2012 were included in this study. All patients were pathologically confirmed to have atelectasis. There were 19 males and 11 females. They ranged in age from 54 to 87 years, with a median age of 71 years. Of all patients, 20 had squamous cell carcinoma, 8 had adenocarcinoma, and 2 had adenosquamous carcinoma; 14 had TNM stage IIIA disease and 16 had stage IIIB disease. Inclusion criteria were: NSCLC patients who were willing to undergo radical three-dimensional conformal radiotherapy; those with lesions detectable by $\mathrm{CT}$ or other imaging modalities; histologically or cytologically confirmed NSCLC; standardized uptake value (SUV) $\geq 2.5$; KPS score $\geq 70$ points; and no pericardial effusion.

\section{Equipment and reagents}

The Biograph 64 PET/CT system (number of slices $=64$; number of detector rings $=39$; Siemens, USA) equipped with the RDS Eclipse cyclotron was used in this study. ${ }^{18} \mathrm{~F}$-fluorodeoxyglucose (FDG) with a radiochemical purity of $>96 \%$ was used.

\section{PET-CT procedure}

All patients were asked to fast for $6 \mathrm{~h}$. Prior to the PET-CT procedure, blood glucose levels were determined. ${ }^{18} \mathrm{~F}$-FDG was then intravenously injected at a dose of $0.15 \mathrm{mCi} / \mathrm{kg}$ in patients whose blood glucose levels were in the normal range. Examinations started $60 \mathrm{~min}$ after injection, which consisted of a spiral CT scan followed by a PET scan during quiet breathing. CT data were used for attenuation correction of PET images. Images were then reconstructed using iterative methods, followed by multi-slice and multi-frame imaging. PET image reconstruction parameters were: slice 
Table 2 GTV obtained from CT and PET-CT images in 30 patients

\begin{tabular}{|c|c|c|c|c|c|c|c|c|c|c|c|c|c|c|c|c|}
\hline GTV $\left(\mathrm{cm}^{3}\right)$ & 1 & 2 & 3 & 4 & 5 & 6 & 7 & 8 & 9 & 10 & 11 & 12 & 13 & 14 & 15 & 16 \\
\hline$C T$ & 301.0 & 76.2 & 134.6 & 131.6 & 102.1 & 146.3 & 98.2 & 178.3 & 153.5 & 165.2 & 167.8 & 214.6 & 156.7 & 108.5 & 101.2 & 245.7 \\
\hline PET-CT & 105.8 & 37.8 & 95.4 & 122.7 & 72.7 & 107.2 & 71.6 & 125.6 & 128.7 & 100.7 & 135.7 & 100.4 & 146.2 & 101.4 & 93.5 & 168.1 \\
\hline GTV $\left(\mathrm{cm}^{3}\right)$ & 17 & 18 & & 19 & 20 & 21 & 22 & 23 & 24 & 25 & & & 27 & 28 & 29 & 30 \\
\hline$\sigma \tau$ & 108.9 & 257.4 & & 92.4 & 101.6 & 106.8 & 163.7 & 144.7 & 114.8 & 105.6 & & & 106.5 & 153.5 & 121.9 & 128.4 \\
\hline PET-CT & 99.4 & 109.1 & & 88.7 & 96.5 & 100.4 & 142.9 & 149.9 & 125.4 & 136.4 & & & 131.4 & 178.7 & 134.1 & 153.8 \\
\hline
\end{tabular}

GTV Gross Tumor Volume, CT Computed Tomography, PET-CT Positron Emission Tomography-Computed Tomography.

thickness for PET image reconstruction $=5 \mathrm{~mm}$, matrix $=$ $168 \times 168$, slice thickness for CT image reconstruction = $5 \mathrm{~mm}$, and cross-sectional resolution $=512 \times 512$. Finally, PET and CT data were transferred to the ADAC Pinnacle3TPS workstation where the data sets were fused automatically.

\section{Target volume delineation}

The fused data sets were transferred to the Elekta TOMCON workstation for delineation of target volumes.

(1) Gross tumor volume (GTV): The GTV refers to clinically or radiologically demonstrable extent and location of tumors, including primary pulmonary lesions and metastatic lymph nodes. Lymph nodes with a short-axis diameter $\geq 1 \mathrm{~cm}$ were considered metastatic lymph nodes. Contours of primary pulmonary lesions and metastatic lymph nodes were first defined based on CT images on lung window ( window width $=1600 \mathrm{Hu}$, window level $=-600 \mathrm{Hu}$ ) and mediastinal window (window width $=400 \mathrm{Hu}$, window level $=20 \mathrm{Hu}$ ) settings to obtain $\mathrm{GTV}_{\mathrm{CT}}$, then based on PET-CT fusion images (also including primary lesions and positive lymph nodes) to obtain $\mathrm{GTV}_{\text {PET-CT. }}$ The threshold of $42 \%$ of maximum SUV (SUVmax) was used for tumor delineation. $\mathrm{GTV}_{\mathrm{CT}}$ and GTV $\mathrm{PET}_{\mathrm{CT}}$ values were calculated automatically on the Elekta TOMCON workstation.

(2) Clinical target volume (CTV): The CTV includes the GTV and the range of subclinical lesions. In this study, a uniform margin of $8 \mathrm{~mm}$ was added around the GTV in adenocarcinoma cases, $6 \mathrm{~mm}$ in squamous cell carcinomas, and 6-8 $\mathrm{mm}$ in adenosquamous carcinoma cases depending on specific situations to form the CTV.

(3) Planning target volume (PTV): The PTV takes into consideration uncertainties caused by physiological displacement of organs, patient movements, and set-up errors that occur during each daily delivery of radiation. Internal target volume (ITV) was used to assess physiological displacement of organs, including the PTV and a margin compensating for daily positioning errors and internal motion of organs. The ideal CTV should result in the highest probability of CTV coverage, thus ensuring exposure of the CTV to maximum prescribed dose irradiation in each fraction of radiation. In this study, the PTV was defined by adding a margin of 10$15 \mathrm{~mm}$ to the GTV.

\section{Delineation of organs at risk (OARs)}

(1)Lungs: automatically delineated on the Elekta TOMCON workstation and then manually modified to exclude the trachea and bronchi.

(2) Heart: delineated from the bottom of the aortic arch to the bottom of the heart.

(3) Esophagus: delineated from the level of the cricoid cartilage to the area above the esophagogastric junction.

(4) Spinal cord: delineated slice by slice after adjusting $\mathrm{CT}$ window width and level to clearly demonstrate the spinal cord.

Table 3 Dose-volume histogram parameters for Plan $_{\text {CT }}$ and Plan

\begin{tabular}{lllll}
\hline Parameter & Plan $_{\text {PET-CT }}$ & Plan $_{\text {CT }}$ & $\boldsymbol{t}$ & $\boldsymbol{P}$ \\
\hline Lungs & & & & \\
$V_{5}(\%)$ & $46.2 \pm 22.3$ & $46.4 \pm 22.5$ & -0.866 & 0.394 \\
$V_{10}(\%)$ & $37.6 \pm 19.1$ & $37.9 \pm 19.6$ & -1.738 & 0.089 \\
$V_{15}(\%)$ & $26.7 \pm 11.9$ & $27.3 \pm 15.4$ & -0.938 & 0.352 \\
$V_{20}(\%)$ & $18.1 \pm 9.6$ & $19.6 \pm 10.2$ & -2.108 & 0.040 \\
$V_{30}(\%)$ & $13.2 \pm 7.4$ & $14.8 \pm 8.5$ & -2.082 & 0.042 \\
MLD (Gy) & $13.1 \pm 4.9$ & $13.9 \pm 5.3$ & -1.918 & 0.062 \\
Heart & & & & \\
$V_{30}(\%)$ & $20.5 \pm 17.4$ & $21.6 \pm 19.2$ & -0.676 & 0.502 \\
MHD (Gy) & $15.8 \pm 9.1$ & $16.7 \pm 10.3$ & -0.659 & 0.512 \\
Esophagus & & & & \\
$V_{50}(\%)$ & $19.6 \pm 15.1$ & $24.5 \pm 18.4$ & -2.326 & 0.029 \\
$V_{55}(\%)$ & $15.4 \pm 13.8$ & $20.7 \pm 17.6$ & -2.366 & 0.022 \\
Dmax (Gy) & $61.2 \pm 10.2$ & $61.7 \pm 12.1$ & -0.083 & 0.936 \\
Spinal cord & & & & \\
Dmax (Gy) & $40.3 \pm 10.9$ & $43.2 \pm 11.5$ & -1.892 & 0.065 \\
\hline Dmax maximum & & & &
\end{tabular}

Dmax maximum dose, MHD Mean Heart Dose, MLD Mean Lung Dose, $V_{x}$ the percentage volume of organ receiving $X$ Gy of dose, MLD Mean Lung Dose, MHD Mean Heart Dose, Dmax maximum dose. 


\section{Radiation treatment planning}

Treatment planning was performed using the Elekta Precise Plan treatment planning system.

The PTV $\mathrm{CT}_{\mathrm{CT}}$ and PTV $\mathrm{PET}_{\text {-CT }}$ were defined by adding a margin of 1.0-1.5 $\mathrm{cm}$ to the $\mathrm{GTV}_{\mathrm{CT}}$ and GTV $\mathrm{GET}_{\text {-CT. }}$ An experienced physician was responsible for designing three-dimensional conformal radiotherapy treatment plans Plan CT $_{\text {and Plan }}$ PET-CT on CT image sets. 95\% of the PTV should be encompassed by the $90 \%$ isodose curve, and the two treatment plans should keep the same beam direction, beam number, gantry angle, and position of the multi-leaf collimator as much as possible. The prescribed dose was 2 Gy per daily fraction, 5 days per week, to a total dose of $60 \mathrm{~Gy}$ in 30 fractions. Dose constraints to OARs were lung $\mathrm{V}_{20}<35 \%$, heart $\mathrm{D}_{1 / 3}<50 \mathrm{~Gy}$, spinal cord Dmax $<45$ Gy, and esophagus Dmax $<60$ Gy.

\section{Main indicators and parameters}

(1) GTV $V_{\mathrm{CT}}$ and GTV $\mathrm{PET}_{\text {-CT }}$ calculated automatically on the workstation;

(2)Dose-volume histogram (DVH) parameters, including mean lung dose (MLD), lung $\mathrm{V}_{5}, \mathrm{~V}_{10}, \mathrm{~V}_{15}$, $\mathrm{V}_{20}$ and $\mathrm{V}_{30}$, mean heart dose (MHD), heart $\mathrm{V}_{30}$, esophageal $V_{50}$ and $V_{55}$, esophageal Dmax, and spinal cord Dmax.

\section{Statistical analysis}

Statistical analysis was performed using SPSS 19.0 software. Comparisons between two groups were performed using the $t$-test. Data were expressed as mean \pm standard deviation (SD). $P<0.05$ was considered statistically significant.

\section{Results}

\section{GTV}

The $\mathrm{GTV}_{\mathrm{CT}}$ and $\mathrm{GTV}_{\mathrm{PET}-\mathrm{CT}}$ had varying degrees of change in all 30 patients, and the changes in $\mathrm{GTV}_{\mathrm{CT}}$ and $\mathrm{GTV}_{\text {PET-CT }}$ exceeded 25\% in 12 (40\%) patients.

The $\mathrm{GTV}_{\mathrm{PET}-\mathrm{CT}}$ decreased in varying degrees compared to the $\mathrm{GTV}_{\mathrm{CT}}$ in 22 patients. Their median $\mathrm{GTV}_{\text {PET-CT }}$ and median $\mathrm{GTV}_{\text {PET-CT }}$ were $111.4 \mathrm{~cm}^{3}$ (range, $37.8 \mathrm{~cm}^{3}-188.7 \mathrm{~cm}^{3}$ ) and $155.1 \mathrm{~cm}^{3}$ (range, 76.2 $\mathrm{cm}^{3}-301.0 \mathrm{~cm}^{3}$ ), respectively, and the former was 43.7 $\mathrm{cm}^{3}$ (28.2\%) less than the latter.

The $G_{T V} V_{\text {PET-CT }}$ increased in varying degrees compared to the $\mathrm{GTV}_{\mathrm{CT}}$ in 8 patients. Their median GTV $_{\text {PET-CT }}$ and median GTV $_{\text {PET-CT }}$ were $144.7 \mathrm{~cm}^{3}$ (range, $125.4 \mathrm{~cm}^{3}-178.7 \mathrm{~cm}^{3}$ ) and $125.8 \mathrm{~cm}^{3}$ (range, $105.6 \mathrm{~cm}^{3}-153.5 \mathrm{~cm}^{3}$ ), respectively, and the former was $18.9 \mathrm{~cm}^{3}(15.0 \%)$ greater than the latter.

The main reason for the decrease in the $\mathrm{GTV}_{\mathrm{PET}-\mathrm{CT}}$ relative to the $\mathrm{GTV}_{\mathrm{CT}}$ is that PET-CT allowed distinguishing tumor tissue from collapsed lung tissue based on their difference in functional metabolism and reducing the target volumes (Figure $1 \mathrm{~A}$ and $\mathrm{B}$ ). In contrast, it was difficult to distinguish the boundaries between incompletely expanded lung tissue and tumor tissue by conventional CT, which resulted in excessive target delineation.
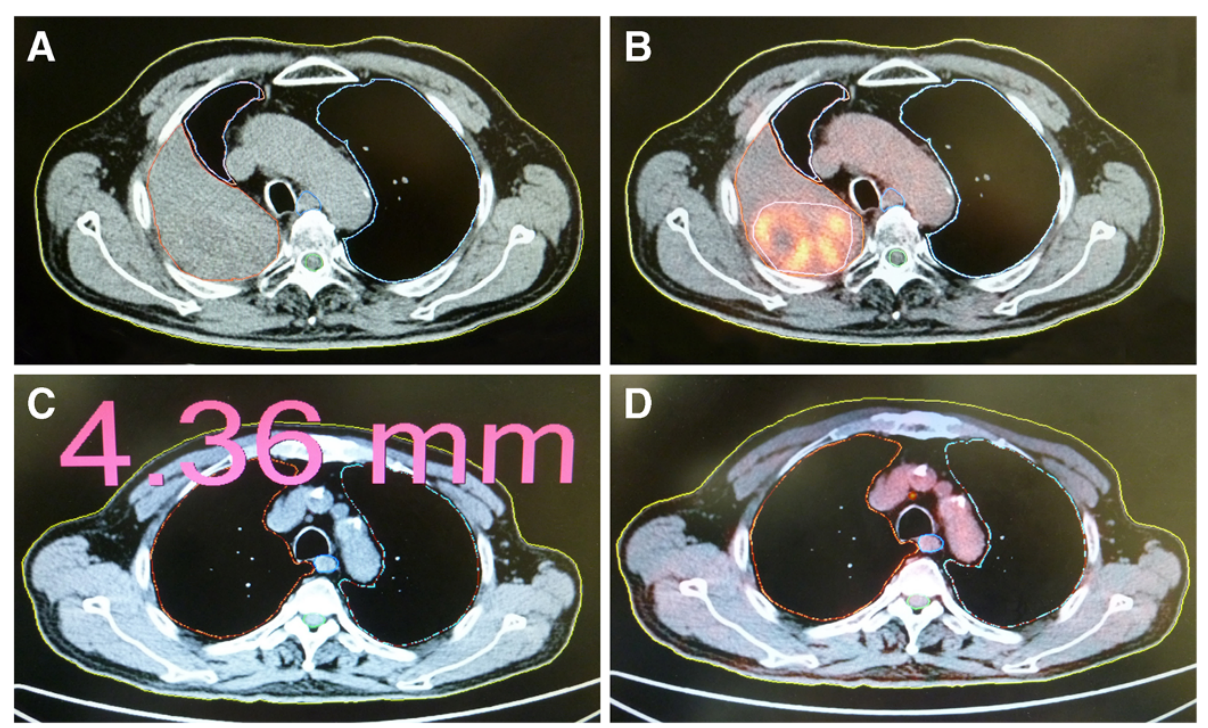

Figure 1 Comparison between conventional CT and PET-CT image. A: By conventional $C T$, it was difficult to distinguish the boundaries between incompletely expanded lung tissue and tumor tissue with consequent excessive target delineation. B: By PET-CT, the areas of high metabolic activity indicated the presence of tumors. The target volume was obviously smaller than that on conventional CT image. C: On conventional CT image, the mediastinal lymph node had a short-axis diameter of $4.36 \mathrm{~mm}$ and was not considered a metastatic lymph node. D: On PET-CT image, the mediastinal lymph node showed high metabolic activity and was considered a metastatic lymph node. 
The main reason for the increase in the GTV $\mathrm{PET}_{\text {-CT }}$ relative to the $\mathrm{GTV}_{\mathrm{CT}}$ is that PET-CT allowed detecting metastatic lymph nodes that could not be identified by conventional $\mathrm{CT}$ and increasing the target volumes (Figure $1 \mathrm{C}$ and $\mathrm{D})$.

\section{OARs}

Compared to Plan $_{\mathrm{CT}}$ parameters, Plan ${ }_{\mathrm{PET}-\mathrm{CT}}$ parameters showed varying degrees of change. The changes in lung $\mathrm{V}_{20}, \mathrm{~V}_{30}$, esophageal $\mathrm{V}_{50}$ and $\mathrm{V}_{55}$ were statistically significant $(P s<0.05$ for all), while the differences in MLD, lung $\mathrm{V}_{5}, \mathrm{~V}_{10}, \mathrm{~V}_{15}$, heart $\mathrm{V}_{30}, \mathrm{MHD}$, esophageal Dmax, and spinal cord Dmax were not statistically significant (PS> 0.05 for all).

\section{Discussion}

Generally, three-dimensional conformal radiotherapy treatment planning is based on CT images; however, the information provided by $\mathrm{CT}$ data often cannot meet the requirements of target volume delineation [7]. In recent years, PET-CT has been increasingly used in clinical practice to delineate the target volumes for radiotherapy of lung cancer. PET-CT has an accuracy superior to that of conventional CT and other imaging modalities. DeniaudAlexandre et al. [8] delineated the GTV in 92 NSCLC patients by PET-CT and found that the $\mathrm{GTV}_{\text {PET-CT }}$ was reduced in $23 \%$ of the patients and increased in $26 \%$ of cases compared to $\mathrm{GTV}_{\mathrm{CT}}$, and 21 patients had a GTV change of $\geq 25 \%$. In this study, we found that all 30 patients had varying degrees of changes in the GTV $\mathrm{PET}_{\text {-CT }}$ and $\mathrm{GTV}_{\mathrm{CT}}$, including 12 (40\%) patients who had a change over $25 \%$. This result is consistent with those reported by DeniaudAlexandre et al. and Ceresoli et al. [9].

Although it is important to meet the requirements of target dose distribution, serious complications of radiation therapy caused by too large irradiated volume or too high dose to OARs should also be avoided. A given radiation treatment plan in which the target irradiation volume and the PTV fit well and the dose is evenly distributed is still unacceptable when the dose to OARs exceeds the tolerable dose of the organ or the irradiated volume of OARs is too large, because the implementation of this treatment plan will cause great damage to normal tissue and serious radiotherapy complications. Bradley et al. [10] contoured the GTV from the CT and PET-CT data sets in 26 NSCLC patients and found that, in three patients with atelectasis, the GTV and PTV obtained from PET-CT images were significantly reduced compared to those obtained from CT images, the MLD decreased from 14.83 Gy to $12.93 \mathrm{~Gy}$, and lung $\mathrm{V}_{20}$ decreased from $25.33 \%$ to $21.33 \%$. They also discovered that the MLD and mean esophageal dose increased with the increase in the GTV in 11 patients whose target volumes increased as a result of additional detection of metastatic lymph nodes. Van Der Wel et al. [11] contoured the target volumes by PET-CT and found that the GTV of the lymph nodes, lung $V_{20}$, MLD, esophageal $V_{45}$ and $V_{55}$ decreased. At the same level of radiation toxicity, radiation dose and tumor control rate were improved. As a result, the efficacy of radiation therapy was enhanced. In the present study, we found

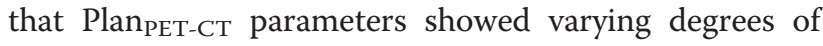
change compared to Plan $_{\mathrm{CT}}$ parameters. The changes in lung $\mathrm{V}_{20}, \mathrm{~V}_{30}$, esophageal $\mathrm{V}_{50}$ and $\mathrm{V}_{55}$ were statistically significant (Ps< 0.05 for all), while the differences in MLD, lung $\mathrm{V}_{5}, \mathrm{~V}_{10}, \mathrm{~V}_{15}$, heart $\mathrm{V}_{30}, \mathrm{MHD}$, esophageal Dmax, and spinal cord Dmax were not significant (Ps> 0.05 for all).

Acute radiation-induced lung injury is a kind of lymphocytic alveolar inflammation caused by direct radiation damage and body's immune response. The severity of lung functional injury after radiotherapy is closely related to the irradiated volume. The dose-volume histogram (DVH) offers a range of physical parameters for the evaluation of radiotherapy-induced lung injury in lung cancer patients after three-dimensional conformal radiotherapy. The $V_{20}$ is currently the most widely used parameter for clinical evaluation of treatment plans. However, the results obtained on factors associated with acute radiation-induced lung injury are different among different studies. In a study involving 99 NSCLC patients performed by Graham et al. [12], univariate analysis showed that the $\mathrm{V}_{20}$ and MLD were closely associated with the development of acute radiation-induced lung injury (grade 2 or higher), and multivariate analysis showed that the $\mathrm{V}_{20}$ was the only independent predictive factor for acute radiation-induced lung injury. This result is consistent with that obtained by Tsujino et al. [13]. In a study conducted by Zhang et al. [14], univariate analysis indicated that the MLD, mean dose to the affected lung, and $V_{20}$ were factors associated with the development of acute radiation-induced lung injury, and multivariate analysis indicated that only the mean dose to the affected lung is the independent risk factor. Studies performed by Hernando et al. [15], Claude et al. [16], and Kim et al. [17] demonstrated that the $V_{30}$ was a factor associated with the development of acute radiationinduced lung injury. A recent study by Wang et al. [18] showed that the $\mathrm{V}_{5}$ is also associated with the development of acute radiation-induced lung injury, suggesting that $\mathrm{V}_{5}$ as a dose-volume constraint should be fully taken into account in designing radiation treatment

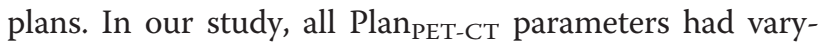
ing degrees of decrease compared to Plan ${ }_{\mathrm{CT}}$ parameters, indicating that delineation of the target volumes for radiotherapy by $\mathrm{PET}-\mathrm{CT}$ can help reduce the incidence of acute radiation-induced lung injury in NSCLC patients. 
Acute radiation-induced esophageal injury usually occurs about two weeks after the start of radiotherapy. In recent years, there have been more and more studies investigating factors associated with the development of esophageal injury in patients undergoing three-dimensional conformal radiotherapy for NSCLC, although the parameters used and the conclusions drawn varied among different studies. Kim et al. [19] suggested that the $\mathrm{V}_{60}$ was an important parameter to predict acute radiation esophagitis (grade 3 or higher). Algara et al. [20] found that the $V_{50}$ was the most valuable predictor. Topkan et al. [21] indicated that the $V_{55}$ was the only relevant dosimetric parameter. More studies indicated that the $V_{55}$ was likely to be the most valuable parameter for predicting acute radiation-induced esophageal injury. Our results indicated that the decrease in esophageal $\mathrm{V}_{55}$ obtained using PlanPET-CT was statistically significant $(\mathrm{p}<0.05)$ compared to that obtained using PlanCT, suggesting that delineation of the target volumes for radiotherapy by PET-CT can help reduce the incidence of acute radiation-induced esophageal injury in NSCLC patients.

Radiation myelitis is a myelopathy that develops following spinal cord exposure to therapeutic radiation [22]. Due to the combined effects of a variety of factors, neuronal degeneration and necrosis occur. The development of radiation myelitis is associated with exposure of normal spinal cord tissue to high-dose radiation $[23,24]$. In our study, spinal cord Dmax obtained using PlanPETCT decreased compared to that obtained using PlanCT, but the difference was not statistically significant. More emphasis should be put on the prevention of radioactive myelitis, and radiation dose to the spinal cord must be strictly controlled during radiotherapy.

Radiation damage to the heart is mainly manifested as ECG abnormalities, especially ischemic ST-T changes. The incidence of heart injury will significantly increase if $1 / 3$ of the heart volume receives $70 \mathrm{~Gy}, 2 / 3$ of the heart volume receives $55 \mathrm{~Gy}$, or the whole heart receives 50 Gy. Our study suggests that PET-CT can help protect from cardiac injury to a certain extent, although the difference was not obvious between the two groups.

\section{Conclusions}

In summary, the results of this study show that PET-CT fusion image is beneficial to target area sketch in patients merged with atelectasis of non-small cell lung cancer, and optimization of precise radiotherapy planning. By lowering the irradiation dose of the surrounding normal lung tissue and avoiding missing the irradiation of target area, the utilization of PET-CT can reduce the probability of occurrence of acute radiation-induced lung injury and acute radiation-induced esophageal injury. Also, the utilization of PET-CT has a protective effect on the heart and spinal cord. It brings the benefits to the lung cancer patients. Therefore, we believe that PET-CT will play more and more important role in radiotherapy of NSCLC.

\section{Abbreviations}

3D-CRT: Three-dimensional conformal radiotherapy; CTV: Clinical target volume; DHV: Dose volume histograms; GTV: Gross tumor volume; ITV: Internal target volume; PTV: Planning target volume; MHD: Mean heart dose; MLD: Mean lung dose; OAR: Organs at risk; SUV: Standardized uptake value.

\section{Competing interests}

The authors declare that they have no competing interests.

\section{Author details}

${ }^{1}$ Department of Radiotherapy, Dalian Central Hospital, Dalian 116033, China. ${ }^{2}$ China Petroleum Central Hospital, Langfang 065000, China. ${ }^{3}$ China Medical University, Shenyang 110001, China.

Received: 30 August 2012 Accepted: 4 February 2013

Published: 18 March 2013

\section{References}

1. Zhang ZJ: Technical capacity and clinical application of PET/CT. Chin Med Equipm J 2007, 28(10):59-60.

2. Chuhr KJH, Kim JH, Yoon DY, Hwang HS, Rho YS: Additional diagnostic value of (18)F-FDG PET-CT in detecting retropharyngeal nodal metastases. Otolaryngol Head Neck Surg 2009, 141(5):633-638.

3. Kang BJ, JH O, Baik JH, Jung SL, Park YH, Chung SK: Incidental thyroid uptake on F-18 FDG PET/CT: correlation with ultrasonography and pathology. Ann Nucl Med 2009, 23(8):729-737.

4. Balogh JB, Caldwell CB, Ung YC, et al: Interobserver variation in contouring gross tumor volume in carcinoma of the lung associated with pneumonitis and atelectasis: the impactof18FDG-hybrid pet fusion. Int J Radiat Oncol Biol Phys 2000, 48:128-129.

5. Hoseok I,Kim K, Kim SJ, Kim IJ, Pak K, Kim H: Prognostic value of metabolic volume measured by F-18 FDG PET-CT in patients with esophageal cancer. Thoracic Cancer 2012, 3:255-261.

6. Wang $\mathrm{DQ}$, Chen $\mathrm{JH}$, Li BS, et al: Influence of FDG PET-CT on the target region and planning of precise and accurate padiotherapy for local advanced non-small cell lung cancer. Chinese J Radiat Oncol 2011, 20:172-173.

7. Zhang QN, Wang DY, Wang XH, Hui TJ, Yang KH, Li Z, Li HY, Guo LY: Nonconventional radiotherapy versus conventional radiotherapy for inoperable non-small-cell lung cancer: a meta-analysis of randomized clinical trials. Thoracic Cancer 2012, 3(3):269-279.

8. Deniaud-Alexandre E, Touboul E, Lerouge D, Grahek D, Foulquier JN, Petegnief $Y$, Grès B, El Balaa H, Keraudy K, Kerrou K, Montravers F, Milleron B, Lebeau B, Talbot JN: Impact of computed tomography and 18Fdeoxyglucose coincidence detection emission tomography image fusion for optimization of conformal radiotherapy in non-small cell lung cancer. Int J Radiat Oncol Biol Phys 2005, 63(5):1432-1441.

9. Ceresoli GL, Cattaneo GM, Castellone P, Rizzos G, Landoni C, Gregorc V, Calandrino R, Villa E, Messa C, Santoro A, Fazio F: Role of computed tomography and 18F-fluoro-deoxyglucose positron emission tomography image fusion in conformal radiotherapy of non-small cell lung cancer a comparison with standard techniques with and without elective nodal irradiation. Tumori 2007, 93(1):88-96.

10. Bradley J, Thorstad WL, Mutic S, et al: Impact of FDG-PET on radiation therapy volume delineation in non-small cell lung cancer. Int J Radial Oncol Biol Phys 2004, 59:78-86.

11. Van Der Wel A, Nijsten S, Hochstenbag M, Lamers R, Boersma L, Wanders R, Lutgens L, Zimny M, Bentzen SM, Wouters B, Lambin P, De Ruysscher D: Increased therapeutic ratio by ${ }^{18}$ FDG-PETCT planning in patients with clinical CT stage N2-N3 Mo non-small-cell lung cancer: a modeling study. Int J Radiat Oncol Biol Phys 2005, 61:649-655.

12. Graham MV, Purdy JA, Emami B, Harms W, Bosch W, Lockett MA, Perez CA: Clinical dose-volume histogram analysis for pneumonitis after 3D treatment for non-small cell lung cancer (NSCLC). Int J Radiat Oncol Biol Phys 1999, 45:323-329.

13. Tsujino K, Hirota S, Endo M, Obayashi K, Kotani Y, Satouchi M, Kado T, Takada Y: Predictive value of dose-volume histogram parameters for 
predicting radiation pneumonitis after concurrent chemoradiation for lung cancer. Int J Radiat Oncol Biol Phys 2003, 55:110-115.

14. Zhang YJ, Li JB, Tian SY, et al: Analysis of radiation-induced lung injury after lung cancer radiotherapy. Chin J Cancer Prevent Treat 2008, 15:1264-1267.

15. Hernando ML, Marks LB, Bentel GC, Zhou SM, Hollis D, Das SK, Fan M, Munley MT, Shafman TD, Anscher MS, Lind PA: Radiation-induced pulmonary toxicity: a dose-volume histogram analysis in 201 patients with lung cancer. Int J Radiat Oncol Biol Phys 2001, 51:650-659.

16. Claude L, Perol D, Ginestet C, Falchero L, Arpin D, Vincent M, Martel I, Hominal S, Cordier JF, Carrie C: A prospective study on radiation pneumonitis following conformal radiation therapy in non-small lung cancer:clinical and dosimetric factors analysis. Radiother Oncol 2004, 71:175-181.

17. Kim TH, Cho KH, Pyo HR, Lee JS, Zo Jl, Lee DH, Lee JM, Kim HY, Hwangbo B, Park SY, Kim JY, Shin KH, Kim DY: Dose-volumetric parameters for predicting severe radiation pneumonitis after three-dimensional conformal radiation therapy for lung cancer. Radiology 2005, 235:208-215.

18. Wang J, Wang P, Pang QS, et al: Clinical and dosimetric factors associated with radiation-induced lung damage in patients with non-small cell lung cancer treated with three-dimentional conformal radiotherapy. Chin J Radiat Oncol 2009, 18:448-451.

19. Kim TH, Cho KH, Pyo HR, Lee JS, Han JY, Zo JI, Lee JM, Hong EK, Choi IJ, Park SY, Shin KH, Kim DY, Kim JY: Dose-volumetric parameters of acute esophageal toxicity in patients with lung cancer treated with threedimensional conformal radiotherapy. Int J Radiat Oncol Biol Phys 2005, 62:995-1002.

20. Algara M, Rodriguez N, Viñals P, Lacruz M, Foro P, Reig A, Quera J, Lozano J, Fernández-Velilla E, Membrive I, Dengra J, Sanz X: Prevention of radiochemotherapy-induced esophagitis with glutamine: results of a pilot study. Int J Radiat Oncol Biol Phys 2007, 69:342-349.

21. Topkan E, Yavuz MN, Onal C, Yavuz AA: Prevention of acute radiationinduced esophagitis with glutamine in non-small cell lung cancer patients treated with radiotherapy: Evaluation of clinical and dosimetric parameters. Lung Cancer 2009, 63:393-399.

22. Nichols Jr RC,Huh SH, Henderson RH, Li Z, Flampouri S, D'Agostino HJ, Cury JD, Pham DC,Mendenhall NP, Hoppe BS: Selective nodal irradiation of regionally advanced non-small-cell lung cancer with proton therapy and IMRT: a dosimetric comparison. Thoracic Cancer 2012, 3:169-174.

23. Gimeno-García AZ, Elwassief A, Paquin SC,Sahai AV: Endoscopic ultrasoundguided fine needle aspiration cytology and biopsy in the evaluation of lymphoma. Endoscopic Ultrasound 2012, 1:17-22.

24. Liu Y, Chen G, Qiu X, Chen J, Zhou Q: The 12th National Lung Cancer Academic Conference in China. Thoracic Cancer 2012, 3:287-288.

\section{doi:10.1186/2049-6958-8-21}

Cite this article as: Yin et al.: Utilization of PET-CT in target volume delineation for three-dimensional conformal radiotherapy in patients with non-small cell lung cancer and atelectasis. Multidisciplinary Respiratory Medicine 2013 8:21.

\section{Submit your next manuscript to BioMed Central and take full advantage of:}

- Convenient online submission

- Thorough peer review

- No space constraints or color figure charges

- Immediate publication on acceptance

- Inclusion in PubMed, CAS, Scopus and Google Scholar

- Research which is freely available for redistribution

Submit your manuscript at www.biomedcentral.com/submit
C Biomed Central 